\title{
First Experience with Chemokine Receptor CXCR4-Targeted PET Imaging of Patients with Solid Cancers
}

\author{
Tibor Vag ${ }^{1}$, Carlos Gerngross ${ }^{1}$, Peter Herhaus ${ }^{2}$, Matthias Eiber ${ }^{1}$, Kathrin Philipp-Abbrederis ${ }^{2}$, Frank-Philipp Graner ${ }^{1}$, \\ Johannes Ettl ${ }^{3}$, Ulrich Keller ${ }^{2,4}$, Hans-Jürgen Wester ${ }^{5}$, and Markus Schwaiger ${ }^{1,5}$ \\ ${ }^{1}$ Clinic of Nuclear Medicine, Klinikum Rechts der Isar, Technische Universität München, Munich, Germany; ${ }^{2} I I I$ Medical Department, \\ Klinikum Rechts der Isar, Technische Universität München, Munich, Germany; ${ }^{3}$ Clinic of Gynecology, Klinikum Rechts der Isar, \\ Technische Universität München, Munich, Germany; ${ }^{4}$ German Cancer Consortium (DKTK) and German Cancer Research \\ Center (DKFZ), Heidelberg, Germany; and ${ }^{5}$ Institute of Pharmaceutical Radiochemistry, Technische Universität München, \\ Garching, Germany
}

CXCR4 is a chemokine receptor that is overexpressed in various human cancers and is involved in tumor metastasis. The aim of this proof-of-concept study was to evaluate a novel CXCR4-targeted PET probe in patients with solid cancers with reported in vitro evidence of CXCR4 overexpression and to estimate its potential diagnostic value. Methods: Twenty-one patients with histologically proven pancreatic cancer, laryngeal cancer, non-small cell lung cancer, prostate cancer, melanoma, breast cancer, hepatocellular carcinoma, glioblastoma, sarcoma, or cancer of unknown primary underwent PET imaging using the novel CXCR4 nuclear probe ${ }^{68} \mathrm{Ga}$-pentixafor. The SUV $_{\max }$ of the liver, spleen, and bone marrow was measured to determine physiologic tracer distribution. For evaluation of tracer accumulation in solid cancers, SUV $\max$ and tumor-tobackground (T/B) ratios were determined in a total of 43 malignant lesions, including 8 primary tumors, 3 locally recurrent tumors, and 32 metastases. When available, the SUV $\max$ of malignant lesions was compared with the corresponding $S_{U} V_{\max }$ measured in routine ${ }^{18}$ F-FDG PET. Results: Moderate tracer accumulation was detectable in the liver, bone marrow, and spleen, with a mean $S_{\text {SUax }}$ of $3.1,3.7$, and 5.6, respectively. By visual interpretation criteria, 9 of 11 primary and locally recurrent tumors were detectable, exhibiting a mean SUV max $_{\text {ax }}$ of 4.7 (range, 2.1-10.9) and a mean T/B ratio of 2.9. Twenty of 32 evaluated metastases were visually detectable (mean SUV max $_{\text {, }}$ 4.5 [range, 3.2-13.8]; mean T/B ratio, 2.8). The highest signal was detected in a patient with non-small cell lung cancer $\left(\mathrm{SUV}_{\max }\right.$, 10.9; T/B ratio, 8.4) and a patient with cancer of unknown primary $\left(\mathrm{SUV}_{\max }, 13.8\right.$; T/B ratio, 8.1). Compared with ${ }^{18} \mathrm{~F}-\mathrm{FDG}$ $\mathrm{PET}$, which was additionally performed in 10 patients, ${ }^{68} \mathrm{Ga}-$ pentixafor PET had a lower SUV max $_{\text {in }}$ in all measured malignant lesions. Conclusion: On the basis of these first observations in a small and heterogeneous patient cohort, the in vitro CXCR4 expression profile of solid cancers and metastases described in the previous literature does not seem to sufficiently depict the in vivo distribution revealed by CXCR4-targeted PET. Moreover, the detectability of solid cancers seems to be generally lower for ${ }^{68} \mathrm{Ga}$-pentixafor than for ${ }^{18} \mathrm{~F}-\mathrm{FDG} \mathrm{PET}$.

Received May 22, 2015; revision accepted Dec. 1, 2015.

For correspondence or reprints contact: Tibor Vag, Clinic of Nuclear Medicine, Klinikum Rechts der Isar, Technische Universität München, Ismaninger Strasse 22, 81675 Munich, Germany.

E-mail: tibor.vag@tum.de

Published online Jan. 14, 2016.

COPYRIGHT (C 2016 by the Society of Nuclear Medicine and Molecular Imaging, Inc.
Key Words: CXCR4; chemokine receptor; positron emission tomography; in vivo imaging; solid cancers

J Nucl Med 2016; 57:741-746

DOI: 10.2967/jnumed.115.161034

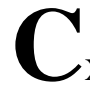

XCR4 is a 7-transmembrane G-coupled receptor belonging to the chemokine receptor family and is expressed by a variety of cells during development and thereafter (1). With its cognate ligand stromal cell-derived factor $1 \alpha(2,3)$, its main role in the hematopoietic system is to control stem cell retention and the homing of hematopoietic cells to the bone marrow (4). In addition to these physiologic roles, CXCR4 has been found to be overexpressed by various human cancers, including breast, lung, colon, and pancreatic cancer; melanoma; and hematopoietic malignancies such as multiple myeloma $(1,5-10)$. Furthermore, a growing body of evidence shows that the CXCR4 stromal cell-derived factor $1 \alpha$ axis plays a crucial role in the mechanism of cancer and metastasis. High levels of stromal cellderived factor $1 \alpha$ in organs and tissues such as lymph nodes, lung, liver, bone, and bone marrow are thought to direct the metastasis of CXCR4-expressing tumor cells $(11,12)$. Concordantly, the level of CXCR4 expression was shown to be higher in metastatic sites than in the primary tumors (13), and changes in CXCR4 signaling have been shown to significantly alter metastatic burden in animal models (14). Accordingly, CXCR4 overexpression has been identified as an adverse prognostic factor in some malignancies, including non-small cell lung cancer (NSCLC) and breast cancer $(6,15)$. Because of its critical role in cancer, CXCR4 has been designated a potential therapeutic target and several CXCR4 inhibitors have been developed, with some of them reaching clinical trials $(2,16)$.

These observations highlight and support the possibility that noninvasive imaging of CXCR4 may become a highly interesting new diagnostic or prognostic imaging biomarker through the in vivo quantification of CXCR4 expression levels in tumors and the identification of aggressive subpopulations such as postchemotherapy patients. In addition, CXCR4 imaging may serve as a tool for monitoring novel CXCR4-targeted treatment options, such as pharmacologic or endoradiotherapeutic interventions.

However, despite the fundamental role of CXCR4 in cancer pathogenesis and metastasis, no clinically suitable method for CXCR4 assessment and in vivo quantification has been approved so far. To meet the clinical need for a highly specific and sensitive 
TABLE 1

${ }^{68} \mathrm{Ga}-$ Pentixafor PET Results for All Patients

\begin{tabular}{|c|c|c|c|c|c|c|c|c|c|}
\hline \multirow[b]{2}{*}{ Type of malignancy } & \multirow{2}{*}{$\begin{array}{c}\text { Before any } \\
\text { therapy }\end{array}$} & \multirow[b]{2}{*}{ Modality } & \multicolumn{3}{|c|}{ Primary tumor } & \multicolumn{4}{|c|}{ Metastases } \\
\hline & & & $\mathrm{SUV}_{\max }$ & $\mathrm{T} / \mathrm{B}$ & VD & $n$ & SUV $_{\max }$ & $\mathrm{T} / \mathrm{B}$ & VD \\
\hline Pancreatic cancer & 0 & PET/CT & $6.2^{*}$ & $2.1^{*}$ & 1 & 0 & - & - & - \\
\hline Pancreatic cancer & 1 & PET/MRI & 4.7 & 1.8 & 1 & 0 & - & - & - \\
\hline Pancreatic cancer & 1 & $\mathrm{PET} / \mathrm{CT}$ & 3.2 & 1.4 & 0 & 0 & - & - & - \\
\hline Laryngeal cancer & 1 & $\mathrm{PET} / \mathrm{CT}$ & 4.5 & 2.3 & 1 & 0 & - & - & - \\
\hline NSCLC & 1 & PET/CT & 5.0 & 3.8 & 1 & 0 & - & - & - \\
\hline NSCLC & 1 & $\mathrm{PET} / \mathrm{CT}$ & 10.9 & 8.4 & 1 & 3 & 3.2 & 1.3 & $0 / 3$ \\
\hline Prostate cancer & 0 & PET/CT & - & - & - & $>5$ & 4.2 & 1.4 & $1 / 5$ \\
\hline Malignant melanoma & 0 & $\mathrm{PET} / \mathrm{CT}$ & - & - & - & 1 & 3.9 & 1.8 & $1 / 1$ \\
\hline Malignant melanoma & 0 & $\mathrm{PET} / \mathrm{CT}$ & - & - & - & 3 & 4.5 & 2.2 & $3 / 3$ \\
\hline Breast cancer & 0 & $\mathrm{PET} / \mathrm{CT}$ & - & - & - & 1 & 5.5 & 3.2 & $1 / 1$ \\
\hline Breast cancer & 1 & $\mathrm{PET} / \mathrm{CT}$ & 3.3 & 3 & 1 & 0 & - & - & - \\
\hline Breast cancer & 1 & PET/CT & 4.8 & 3.2 & 1 & $>5$ & 3.2 & 1.6 & $3 / 5$ \\
\hline $\mathrm{HCC}$ & 1 & PET/MRI & 5.0 & 1.9 & 1 & - & - & - & - \\
\hline $\mathrm{HCC}$ & 0 & PET/MRI & $2.4^{*}$ & $0.2^{*}$ & 0 & 1 & 2.4 & 1.5 & $0 / 1$ \\
\hline Glioblastoma & 0 & PET/MRI & $2.1^{*}$ & $1.9^{*}$ & 1 & 0 & - & - & - \\
\hline Sarcoma & 0 & PET/MRI & - & - & - & 1 & 2.1 & 2.4 & $1 / 1$ \\
\hline Sarcoma & 0 & $\mathrm{PET} / \mathrm{CT}$ & - & - & - & 1 & 2.4 & 3.0 & $1 / 1$ \\
\hline Sarcoma & 0 & PET/MRI & - & - & - & $>5$ & 5.3 & 4.0 & $5 / 5$ \\
\hline Sarcoma & 0 & $\mathrm{PET} / \mathrm{CT}$ & - & - & - & 1 & 5.0 & 3.2 & $1 / 1$ \\
\hline Sarcoma & 0 & $\mathrm{PET} / \mathrm{CT}$ & - & - & - & 3 & 3.2 & 1.5 & $1 / 3$ \\
\hline CUP & 1 & PET/CT & - & - & - & 2 & 13.8 & 8.1 & $2 / 2$ \\
\hline Mean or quantity & $9 / 21$ & $15 / 21$ & 4.7 & 2.9 & $9 / 11$ & 32 & 4.5 & 2.8 & $20 / 32$ \\
\hline $\begin{array}{l}\text { *Local recurrence of } p \\
\text { VD }=\text { visual detectabil } \\
S U V_{\max } \text { and } T / B \text { data }\end{array}$ & $\begin{array}{l}\text { y tumors af } \\
\mathrm{ACC}=\text { hep } \\
\text { nean. Maxir }\end{array}$ & $\begin{array}{l}\text { erapy. } \\
\text { lular carci } \\
\text { of } 5 \text { metas }\end{array}$ & ; CUP = & $x_{0}$ & sengen & ary. & more & . & \\
\hline
\end{tabular}

tool for in vivo CXCR4 assessment and quantification, ${ }^{68} \mathrm{Ga}$-pentixafor (formerly known as ${ }^{68} \mathrm{Ga}$-CPCR4.2), a CXCR4-targeted high-affinity nuclear probe, has been developed and evaluated in small cell lung cancer models for PET by Wester's group $(17,18)$. Proof-of-concept investigations on lymphoma-xenografted animal models and the first investigations on patients with hematologic malignancies confirmed high tracer accumulation in tumors, low uptake in background, and concomitantly fast tracer clearance from nontargeted tissues $(18,19)$. In addition, whole-body dosimetry studies on humans revealed excellent pharmacokinetics and thus low radiation burden to patients (20). Subsequent PET investigations on mice xenografted with human CXCR4-positive multiple myeloma cell lines and patients with advanced multiple myeloma provided high-contrast images with higher detection rates for multiple myeloma manifestations than can be achieved with standard ${ }^{18} \mathrm{~F}$ FDG PET/CT scans (21).

On the basis of these highly encouraging data, we studied the use of ${ }^{68} \mathrm{Ga}$-pentixafor PET in patients with solid cancers and here present our first results. We selected malignancies with previously reported in vitro evidence of CXCR4 overexpression to evaluate whether this imaging modality might be of diagnostic value in CXCR4-expressing cancers. Specifically, we intended to correlate tracer accumulation with reported in vitro expression patterns.
The signal intensities of ${ }^{68} \mathrm{Ga}$-pentixafor PET in physiologic and tumor tissue were assessed by $\mathrm{SUV}_{\max }$ measurements and were compared with those of ${ }^{18} \mathrm{~F}$-FDG PET, when available.

\section{MATERIALS AND METHODS}

\section{Patients}

Between 2013 and 2014, 21 patients (mean age, 63 y; range, 55-85 y) with histologically proven solid cancers underwent either ${ }^{68} \mathrm{Ga}$-pentixafor PET/CT or ${ }^{68} \mathrm{Ga}$-pentixafor PET/MR. Detailed patient characteristics, including cancer type and therapeutic status, are given in Table 1. Ten patients additionally underwent a diagnostic ${ }^{18}$ F-FDG PET/CT study for staging purposes within $2 \mathrm{wk}$ after ${ }^{68} \mathrm{Ga}$-pentixafor imaging. No therapy was performed in the interim.

${ }^{68} \mathrm{Ga}$-pentixafor was synthesized and administered according to the requirements of the German Medicinal Products Act (Arzneimittelgesetz $\$ 132 b$ ) and with the approval of the responsible local regulatory authority (Regierung von Oberbayern). Before the investigation began, all patients gave written informed consent to participate. The data analysis was approved by the responsible local ethics committees.

\section{Synthesis of ${ }^{68} \mathrm{Ga}-$ Pentixafor}

${ }^{68} \mathrm{Ga}$-pentixafor was synthesized using a fully automated, good-manufacturing-practice-compliant procedure on a 


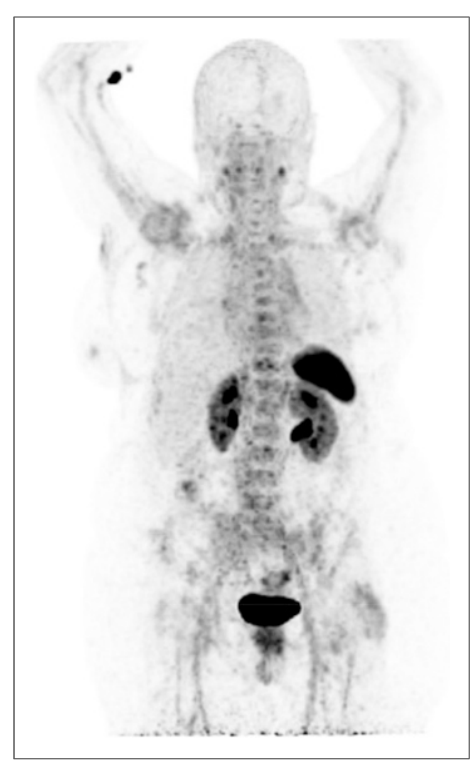

FIGURE 1. Maximum-intensityprojection PET image after tumor excision demonstrates physiologic distribution of ${ }^{68} \mathrm{Ga}$-pentixafor. Highest physiologic uptake is observed in urinary tract (because of renal excretion) and in spleen. Tracer accumulation at right elbow reflects injection site. Medical Solutions).

For PET/CT, a low-dose CT scan (26 mAs, 120 kV, 5-mm slice thickness) from the base of the skull to the mid thigh was acquired for attenuation correction, followed by the PET acquisition and the diagnostic CT acquisition (240 mAs, $120 \mathrm{kV}, 5-\mathrm{mm}$ slice thickness) in the portal venous phase. The injected activity was $147-275 \mathrm{MBq}$ for ${ }^{68} \mathrm{Ga}$-pentixafor and ${ }_{198}-359 \mathrm{MBq}$ for ${ }^{18} \mathrm{~F}-\mathrm{FDG}$. The PET acquisition began at a mean of $62 \mathrm{~min}$ after ${ }^{68} \mathrm{Ga}$-pentixafor injection and $75 \mathrm{~min}$ after ${ }^{18} \mathrm{~F}$-FDG injection. All scans were acquired in 3-dimensional mode at 3 min per bed position. The images were reconstructed using attenuation-weighted ordered-subsets expectation maximization (4 iterations, 8 subsets) followed by a smoothing gaussian filter ( $5 \mathrm{~mm}$ in full width at half maximum).

For PET/MR, the acquisition began at a mean of 72 min after ${ }^{68} \mathrm{Ga}-$ pentixafor injection. A coronal 2-point Dixon 3-dimensional volumetric interpolated $\mathrm{T} 1$-weighted sequence was performed to generate attenuation maps (22), followed by a dedicated diagnostic sequence for the type of malignancy being examined. The PET data were acquired simultaneously with the MR data in 3-dimensional mode at 3 min per bed position.

\section{Image Analysis}

All ${ }^{68} \mathrm{Ga}$-pentixafor PET/CT and PET/MR images were interpreted by a board-certified nuclear medicine physician and a board-certified radiologist in a binary fashion. Lesions were defined as visually detectable if they could be seen by both reviewers on the PET images. Semiquantitative analysis with determination of $\mathrm{SUV}_{\max }$ involved drawing regions of interest around the primary tumor (in the case of preoperative imaging) and around the metastases (when detectable). If patients had more than 5 metastases, the five with the highest visual uptake were analyzed. The analysis included those lesions that either were histologically verified or were unambiguously malignant because of their morphologic characteristics and dynamic behavior on follow-up. To reduce partial-volume effects, lesions smaller than $10 \mathrm{~mm}$ in diameter were excluded from the analysis. For patients who underwent imaging with both ${ }^{18} \mathrm{~F}-\mathrm{FDG}$
PET/CT and ${ }^{68} \mathrm{Ga}$-pentixafor PET/CT, analysis of the two sets of images was performed analogously. $\mathrm{SUV}_{\max }$ was compared between the ${ }^{18} \mathrm{~F}-\mathrm{FDG}$ and ${ }^{68} \mathrm{Ga}$-pentixafor PET images and was tested for a correlation. With reference to Drzezga et al. (22), we did not differ between the $\mathrm{SUV}_{\max }$ of PET/MR and PET/CT images, assuming that the semiquantitative values of the two modalities would be comparable.

\section{Statistics}

Statistical analysis was performed using MedCalc, version 10.2. We used the Mann-Whitney $U$ test to determine the significance of differences in variables between patient subgroups, and we used the Spearman rank test to check for a correlation in $\mathrm{SUV}_{\max }$ between ${ }^{68} \mathrm{Ga}$-pentixafor PET and ${ }^{18} \mathrm{~F}$-FDG PET.

\section{RESULTS}

\section{Patient Characteristics}

We examined 21 patients with histologically proven solid malignancies: pancreatic cancer, laryngeal cancer, NSCLC, prostate cancer, melanoma, breast cancer, hepatocellular carcinoma, glioblastoma, sarcoma, and cancer of unknown primary (Table 1). Of the 9 patients who had not received therapy before imaging, a primary tumor was present in eight. Of the 12 patients who had received therapy (chemotherapy, radiotherapy, or surgery), the primary tumor had locally recurred in three. One patient had CUP syndrome for which imaging had been unable to identify the primary tumor. Evidence of metastases was seen in 13 patients, three of whom showed more than 5 metastases each.

\section{Physiologic Tracer Accumulation}

A moderate accumulation of ${ }^{68} \mathrm{Ga}$-pentixafor occurred in the liver and bone marrow, with a mean $\mathrm{SUV}_{\text {max }}$ of 3.1 (range, 1.5-4.1) and 3.7 (range, 1.52-6.2), respectively. The spleen demonstrated the highest mean ${ }^{68} \mathrm{Ga}$-pentixafor positivity (mean $\mathrm{SUV}_{\max }, 5.6$; range, 2.5-10.5) (Fig. 1). There was no significant correlation in $\mathrm{SUV}_{\max }$ between treated and nontreated patients for any organ.

\section{${ }^{68}$ Ga-Pentixafor Accumulation in Solid Tumors}

Forty-three malignant lesions were evaluated in 21 patients (8 primary tumors, 3 locally recurrent tumors, and 32 metastases; Table 1). By visual interpretative criteria, 9 of 11 primary and locally recurrent tumors were detectable, exhibiting a mean $\mathrm{SUV}_{\max }$ of 4.7 (range, 2.1-10.9) and a mean tumor-to-background (T/B) ratio of 2.9 (range, 0.2-8.4). The highest $\mathrm{SUV}_{\max }, 10.9$, was measured in a patient with NSCLC. Twenty of 32 evaluated metastases were visually detectable (mean $\mathrm{SUV}_{\text {max }}$, 4.5 [range, 3.2-13.8]; mean T/B ratio, 2.8 [range 1.3-8.1]). The highest $\mathrm{SUV}_{\max }, 13.8$, was in the cervical metastases of the patient with cancer of unknown primary. The Spearman test revealed a low correlation between $\mathrm{SUV}_{\max }$ and number of lesions per patient $(r=0.3)$.

\section{Comparison of ${ }^{68} \mathrm{Ga}$-Pentixafor PET with ${ }^{18} \mathrm{~F}-\mathrm{FDG}$ PET}

${ }^{18} \mathrm{~F}$-FDG PET had been performed within 2 wk after ${ }^{68} \mathrm{Ga}-$ pentixafor PET for 10 patients, with a total of 27 lesions evaluated (2 primary tumors and 25 metastases) (Table 2). All 27 lesions were visually detectable with ${ }^{18} \mathrm{~F}$-FDG, but only 19 with ${ }^{68} \mathrm{Ga}$-pentixafor. Semiquantitative analysis revealed that $\mathrm{SUV}_{\max }$ and T/B ratio in all measured lesions were significantly higher for ${ }^{18} \mathrm{~F}-\mathrm{FDG}$ than for ${ }^{68}$ Ga-pentixafor (Figs. 2 and 3). Accordingly, the mean $\mathrm{SUV}_{\max }$ of all lesions was significantly higher on ${ }^{18} \mathrm{~F}-\mathrm{FDG}$ images than on ${ }^{68} \mathrm{Ga}$-pentixafor images $\left(\mathrm{SUV}_{\max }, 13.8\right.$ vs. $\left.5.0 ; P>0.001\right)$. Details of the per-patient analysis are presented in Table 2 . The Spearman test revealed a low correlation in $\mathrm{SUV}_{\max }$ between ${ }^{18} \mathrm{~F}$-FDG PET and ${ }^{68} \mathrm{Ga}$-pentixafor PET $(r=0.3 ; P=0.99)$. 
TABLE 2

Comparison of ${ }^{68} \mathrm{Ga}$-Pentixafor and ${ }^{18} \mathrm{~F}-\mathrm{FDG}$ PET Results in the Patients Who Underwent Both

\begin{tabular}{|c|c|c|c|c|c|c|c|}
\hline \multirow{2}{*}{$\begin{array}{c}\text { Type of } \\
\text { malignancy }\end{array}$} & \multirow[b]{2}{*}{ Mets $(n)$} & \multicolumn{3}{|c|}{${ }^{68} \mathrm{Ga}$-pentixafor } & \multicolumn{3}{|c|}{${ }^{18} \mathrm{~F}-\mathrm{FDG}$} \\
\hline & & $S U V_{\text {max }}$ & T/B & VD & $\mathrm{SUV}_{\max }$ & $\mathrm{T} / \mathrm{B}$ & VD \\
\hline NSCLC & 3 & 5.2 & 2.6 & $1 / 4$ & 29.3 & 11.6 & $4 / 4$ \\
\hline Melanoma & 3 & 4.5 & 2.2 & $3 / 3$ & 17.7 & 10.2 & $3 / 3$ \\
\hline Melanoma & 1 & 3.9 & 1.8 & $1 / 1$ & 11.1 & 5.5 & $1 / 1$ \\
\hline Breast cancer & 1 & 5.5 & 3.2 & $1 / 1$ & 7.8 & 4.3 & $1 / 1$ \\
\hline Breast cancer* & 5 & 3.5 & 1.5 & $3 / 6$ & 9.2 & 4.6 & $6 / 6$ \\
\hline Sarcoma & 1 & 2.1 & 2.6 & $1 / 1$ & 2.4 & 3.0 & $1 / 1$ \\
\hline Sarcoma & $>5$ & 5.3 & 5.0 & $5 / 5$ & 6.7 & 4.0 & $5 / 5$ \\
\hline Sarcoma & 1 & 5.0 & 3.2 & $1 / 1$ & 6.3 & 3.3 & $1 / 1$ \\
\hline Sarcoma & 3 & 3.2 & 1.5 & $1 / 3$ & 11.1 & 5.3 & $3 / 3$ \\
\hline CUP & 2 & 13.8 & 8.1 & $2 / 2$ & 26.9 & 14.2 & $2 / 2$ \\
\hline
\end{tabular}

*Lesions for which primary tumor was present.

Mets = metastases; VD = visual detectability; CUP = cancer of unknown primary.

SUV $_{\max }$ and T/B data are mean. Maximum of 5 metastases was recorded if patient presented with more than 5 metastases. Overall, 27 lesions were evaluated (2 primary tumors and 25 metastases).

\section{DISCUSSION}

CXCR4 is a key chemokine receptor involved in many processes during tumorigenesis and critical for cancer dissemination (1,5-10).

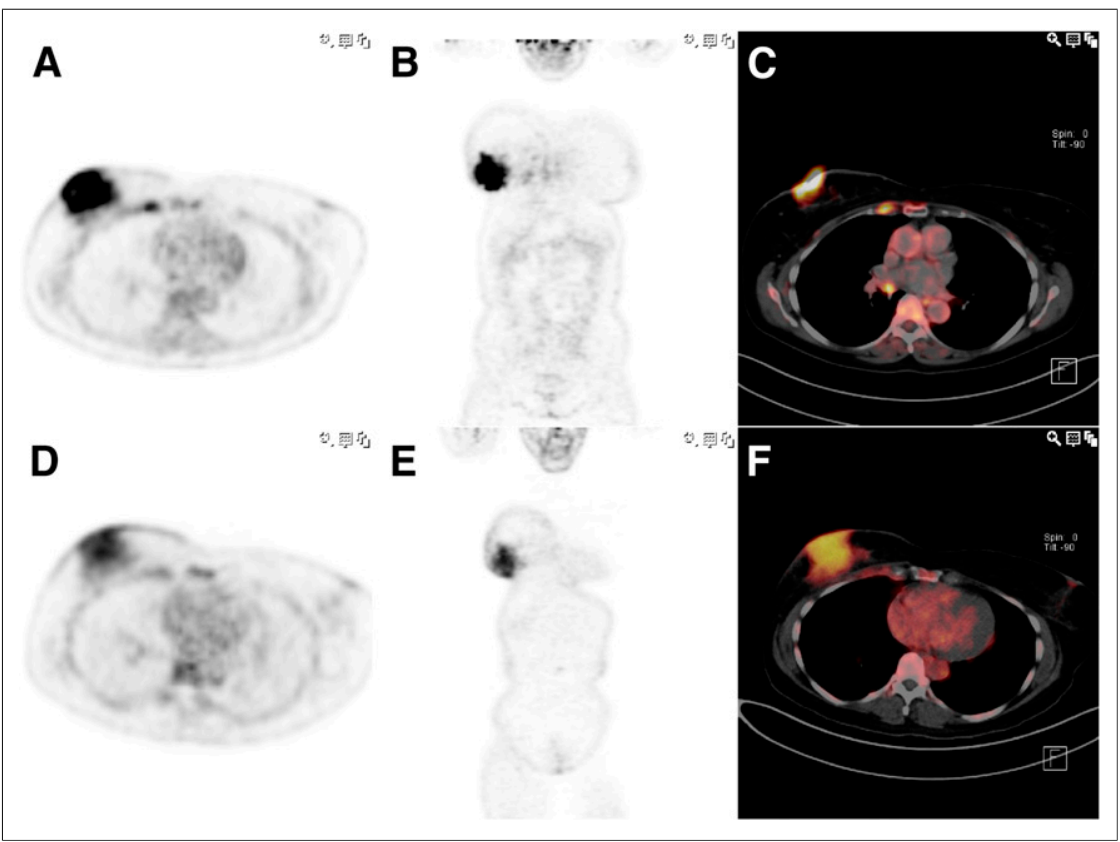

FIGURE 2. 50-y-old patient with histologically proven invasive ductal carcinoma of right breast. Tumor demonstrates intensive uptake on transversal (A), coronal (B), and fusion (C) ${ }^{18} \mathrm{~F}$-FDG PET/CT images. In comparison, uptake is significantly lower on transversal $(D)$, coronal $(E)$, and fusion $(F)^{68} \mathrm{Ga}-$ pentixafor PET/CT images.
In this study, we estimated the diagnostic potential of CXCR4targeted PET imaging in solid cancers for which in vitro evidence of CXCR4 overexpression has been reported (Table 1). For example, significant CXCR4 overexpression has been demonstrated in pancreatic cancer cells compared with normal tissue (23), and high-intensity staining for CXCR4 has been observed in chondrosarcomas through immunohistochemistry, with the most pronounced expression profile being in high-grade sarcomas. Similarly, the aggressiveness of a glioblastoma seems to correlate with its CXCR4 expression pattern (25), and a high accumulation of CXCR4 has been reported to be especially prevalent in breast carcinomas of high metastatic potential $(5,6)$. In melanoma and NSCLC, the detection of high CXCR4 expression has even led to the preclinical development of antagonists, with promising results $(26,27)$.

Interestingly, despite the reported in vitro evidence of CXCR4 overexpression in these solid cancers, ${ }^{68} \mathrm{Ga}$-pentixafor positivity varied significantly between different tumor entities, with an $\mathrm{SUV}_{\max }$ of 10 being exceeded in only 2 patients (one who had NSCLC with a T/B ratio of 8.4 and one who had cervical metastases from cancer of unknown primary with a T/B ratio of 8.1). All other solid malignancies, including pancreatic carcinoma, laryngeal carcinoma, hepatocellular carcinoma, melanoma, breast cancer, glioblastoma, and sarcoma, demonstrated low to modeate ${ }^{68} \mathrm{Ga}$-pentixafor positivity. An accumulation of ${ }^{68} \mathrm{Ga}$-pentixafor was visually detectable in only 29 of 43 cancer lesions - both primary tumors and metastases.

Different reasons might account for the discordance between the high CXCR4 expression profile described in the literature and our current findings. The level of CXCR4 expression assessed by either transcript or whole-cell protein level analysis is not necessarily representative of the CXCR4 expression level on the cell surface $(10,28)$. Shim et al., for example, demonstrated that CXCR4 expression in lymph node metastases of breast cancer patients occurs predominantly in the cytoplasm (28). Because ${ }^{68} \mathrm{Ga}$-pentixafor PET binds to membrane-associated chemokine receptors, a significant discordance might occur between CXCR4 expression profiles determined by transcript or whole-cell protein level analysis and by in vivo quantification of CXCR4 using PET probes. Further relevant factors in this setting might involve the overexpression of CXCR4 on cancer stem cells, which are believed to represent a drug-resistant cell population that can survive even chemotherapy (29-32). Interestingly, the number of cancer stem cells seems to differ with the molecular subtype of the tumor (33) and is believed to correlate with tumor aggressiveness and metastatic potential.

We compared the diagnostic potential of ${ }^{68} \mathrm{Ga}$-pentixafor PET with that of ${ }^{18} \mathrm{~F}-\mathrm{FDG}$ PET by evaluating the 10 of 21 patients with solid malignancies who underwent imaging with both modalities. All 27 of the evaluated malignant lesions within this subpopulation were visually detectable on ${ }^{18} \mathrm{~F}$-FDG PET, compared with only 19 on ${ }^{68} \mathrm{Ga}$-pentixafor PET. Moreover, $\mathrm{SUV}_{\max }$ in all lesions was higher on ${ }^{18} \mathrm{~F}-\mathrm{FDG}$ PET than on ${ }^{68} \mathrm{Ga}$-pentixafor PET $\left(\mathrm{SUV}_{\max }, 13.8\right.$ vs. 


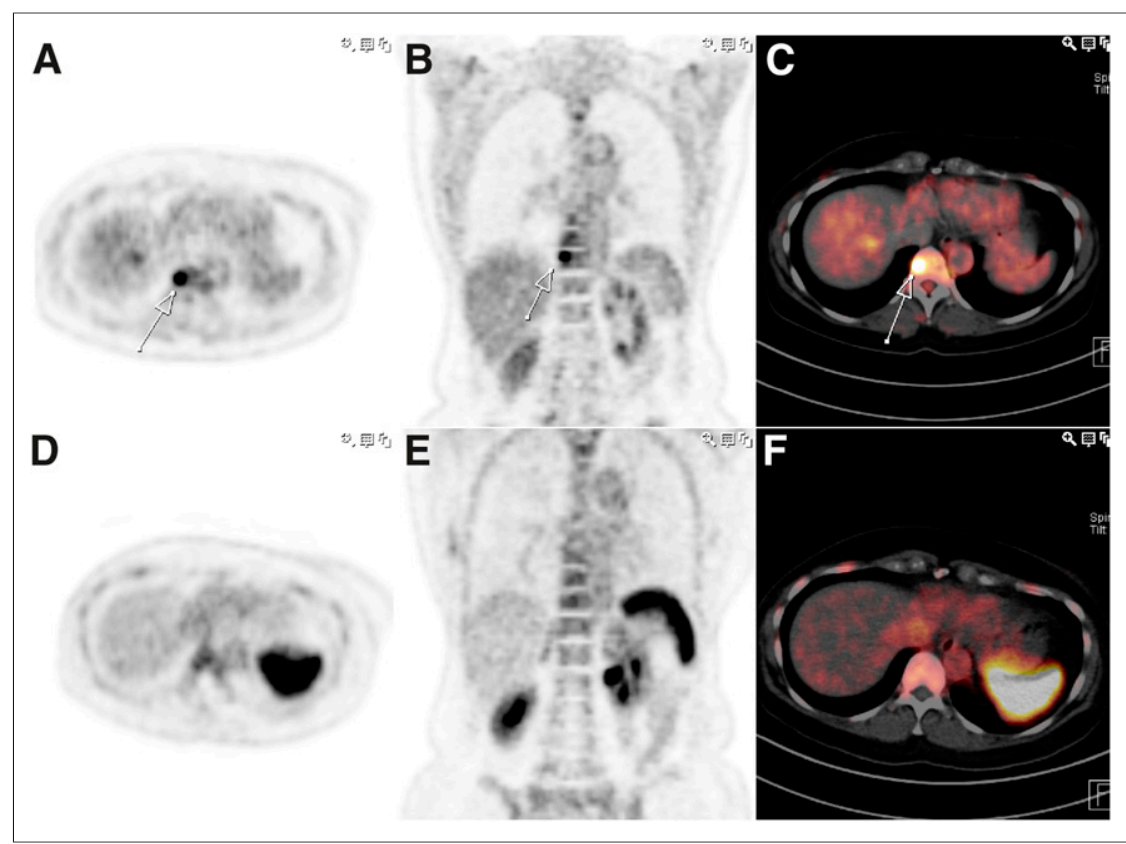

FIGURE 3. 50-y-old patient with invasive ductal carcinoma of right breast and diagnosis of bone metastasis (arrows). Metastasis demonstrates intensive focal uptake on transversal (A), coronal (B), and fusion (C) ${ }^{18} \mathrm{~F}-\mathrm{FDG}$ PET/CT images. In comparison, uptake is not visually detectable on transversal $(\mathrm{D})$, coronal $(\mathrm{E})$, and fusion $(\mathrm{F}){ }^{68} \mathrm{Ga}$-pentixafor PET/CT images.

5.0; $P<0.001)$. Our results indicate that the detectability of solid cancers may generally be lower on ${ }^{68} \mathrm{Ga}$-pentixafor PET than on ${ }^{18} \mathrm{~F}$-FDG PET. These preliminary findings indicate that, for use as a standard oncologic tool, CXCR4-targeted PET does not seem as suitable as ${ }^{18} \mathrm{~F}$-FDG PET. However, the potential of CXCR4-targeted PET might be found in certain specialized applications yet to be elucidated. Several other receptor-binding PET imaging probes already in routine clinical use are tailored to specific tumor types and characteristics. Apart from the often-used somatostatin receptor ligands DOTATATE, DOTATOC, DOTANOC, and HA-DOTATATE for imaging neuroendocrine tumors with low proliferation rates, ligands that bind with high affinity to prostate-specific membrane antigen have been proven to significantly enhance the specificity of prostate cancer imaging (34). Similarly, imaging of CXCR4 might be valuable in only certain subtypes of solid tumors. For example, the benefit of CXCR4 PET imaging in a subset of multiple myeloma patients was recently demonstrated by Philipp-Abbrederis et al., who found specificity and contrast to be superior to ${ }^{18}$ F-FDG (21).

Future efforts should focus on characterization of additional cancer types in which CXCR4 imaging might demonstrate a diagnostic benefit. Besides having the potential to allow highspecificity imaging in certain cancer types, the CXCR4 expression profile would be highly interesting when considering ${ }^{68} \mathrm{Ga}$-pentixafor as a selection marker for CXCR4-directed treatment. ${ }^{177} \mathrm{Lu}$ - or ${ }^{90}$ Y-coupled pentixafor analogs could become attractive radiopharmaceuticals for a theranostic approach, with ${ }^{68} \mathrm{Ga}$-pentixafor PET being used as a marker for patient selection.

The only moderate ${ }^{68} \mathrm{Ga}$-pentixafor positivity of the liver and the bone marrow demonstrated in this study might indicate a favorable side effect profile. The significance of the high variance in splenic tracer uptake, however, has to be further elucidated.

One major limitation of the current study design is the heterogeneity of the patient cohort and the low number of patients per tumor entity. We included patients with various types of malignancies and various stages of disease. A further limitation is the lack of immunohistochemical cross validation. However, we intended to provide a cross section of different cancer entities that have been described in the literature as having considerable CXCR4 expression. The general imaging characteristics of ${ }^{68} \mathrm{Ga}$-pentixafor, such as high specificity of binding, low nonspecific binding, and low background uptake, have already been demonstrated in first patient studies (21). Consequently, our study can serve only as a first impression on the suitability of ${ }^{68} \mathrm{Ga}$-pentixafor PET imaging for patients with the solid tumors listed in Table 1. Such an approach is plausible, considering that experience with this new target and tracer is extremely limited and the areas of application still need to be found. For example, on the basis of the observed findings, examination of NSCLC patients with CXCR4-targeted tracers might be worth investigating. Moreover, metastases from cancer of unknown primary exhibited the highest tracer uptake of all examined lesions, suggesting a potential diagnostic benefit in the detection of the primary cancer. For certain tumor entities, dedicated prospective clinical studies that include correlations with biopsied tumor samples have been initiated.

\section{CONCLUSION}

Our data demonstrate the feasibility of ${ }^{68} \mathrm{Ga}$-pentixafor for PET imaging of solid malignancies. The cancers seemed to exhibit a heterogeneous ${ }^{68} \mathrm{Ga}$-pentixafor accumulation that was lower than that of ${ }^{18} \mathrm{~F}$-FDG. Moreover, the previously described in vitro CXCR4 expression profiles of solid cancers and metastases do not seem to sufficiently depict the in vivo distribution revealed by CXCR4-targeted PET, making further research necessary. However, once the areas of pentixafor imaging are more clearly defined, PET imaging of CXCR4 may prove a valuable modality either as a stand-alone diagnostic tool or in combination with ${ }^{18} \mathrm{~F}$-FDG PET; that is, when considering ${ }^{68} \mathrm{Ga}$-pentixafor for monitoring CXCR4-directed pharmacologic or endoradiotherapeutic treatment.

\section{DISCLOSURE}

The costs of publication of this article were defrayed in part by the payment of page charges. Therefore, and solely to indicate this fact, this article is hereby marked "advertisement" in accordance with 18 USC section 1734. This work received support from the ERC grant MUMI, from the Deutsche Forschungsgemeinschaft (DFG) under grant agreement SFB 824, and from the German Jose Carreras Leukemia Foundation (grant R11/18). Hans-Jürgen Wester is the CEO of Scintomics, the distributor of pentixafor. No other potential conflict of interest relevant to this article was reported. 


\section{REFERENCES}

1. Weiss ID, Jacobson O. Molecular imaging of chemokine receptor CXCR4. Theranostics. 2013;3:76-84.

2. George GPC, Stevens E, Åberg O, et al. Preclinical evaluation of a CXCR4specific ${ }^{68} \mathrm{Ga}$-labelled TN14003 derivative for cancer PET imaging. Bioorg Med Chem. 2014;22:796-803.

3. Zlotnik A. Chemokines and cancer. Int J Cancer. 2006;119:2026-2029.

4. Lapidot T, Dar A, Kollet O. How do stem cells find their way home? Blood. 2005;106:1901-1910.

5. Hung CS, Su HY, Liang HH, et al. High-level expression of CXCR4 in breast cancer is associated with early distant and bone metastases. Tumour Biol. 2014;35: 1581-1588.

6. Yang P, Liang SX, Huang WH, et al. Aberrant expression of CXCR4 significantly contributes to metastasis and predicts poor clinical outcome in breast cancer. Curr Mol Med. 2014;14:174-184.

7. Gao Y, Li C, Nie M, et al. CXCR4 as a novel predictive biomarker for metastasis and poor prognosis in colorectal cancer. Tumour Biol. 2014;35:4171-4175.

8. Liu CF, Liu SY, Min XY, et al. The prognostic value of CXCR4 in ovarian cancer: a meta-analysis. PLoS One. 2014;9:e92629.

9. Maréchal R, Demetter P, Nagy N, et al. High expression of CXCR4 may predict poor survival in resected pancreatic adenocarcinoma. Br J Cancer. 2009;100:1444-1451.

10. Teicher BA, Fricker SP. CXCL12 (SDF-1)/CXCR4 pathway in cancer. Clin Cancer Res. 2010;16:2927-2931.

11. Libura J, Drukala J, Majka M, et al. CXCR4-SDF-1 signaling is active in rhabdomyosarcoma cells and regulates locomotion, chemotaxis, and adhesion. Blood. 2002;100:2597-2606.

12. Furusato B, Mohamed A, Uhlén M, Rhim JS. CXCR4 and cancer. Pathol Int. 2010;60:497-505.

13. Müller A, Homey B, Soto H, et al. Involvement of chemokine receptors in breast cancer metastasis. Nature. 2001;410:50-56.

14. Smith MCP, Luker KE, Garbow JR, et al. CXCR4 regulates growth of both primary and metastatic breast cancer. Cancer Res. 2004;64:8604-8612.

15. Spano JP, Andre F, Morat L, et al. Chemokine receptor CXCR4 and early-stage non-small cell lung cancer: pattern of expression and correlation with outcome. Ann Oncol. 2004;15:613-617.

16. Debnath B, Xu S, Grande F, Garofalo A, Neamati N. Small molecule inhibitors of CXCR4. Theranostics. 2013;3:47-75.

17. Demmer O, Gourni E, Schumacher U, Kessler H, Wester HJ. PET imaging of CXCR4 receptors in cancer by a new optimized ligand. ChemMedChem. 2011;6: 1789-1791.

18. Gourni E, Demmer O, Schottelius M, et al. PET of CXCR4 expression by a ${ }^{68} \mathrm{Ga}-$ labeled highly specific targeted contrast agent. J Nucl Med. 2011;52:1803-1810.

19. Wester HJ, Keller U, Schottelius M, et al. Disclosing the CXCR4 expression in lymphoproliferative diseases by targeted molecular imaging. Theranostics. 2015;5: 618-630.
20. Herrmann K, Lapa C, Wester HJ, et al. Biodistribution and radiation dosimetry for the novel chemokine receptor CXCR4 targeting probe ${ }^{68} \mathrm{Ga}$-pentixafor. $J$ Nucl Med. 2015;56:410-416.

21. Philipp-Abbrederis K, Herrmann K, Knop S, et al. In vivo molecular imaging of chemokine receptor CXCR4 expression in patients with advanced multiple myeloma. EMBO Mol Med. 2015;7:477-487.

22. Drzezga A, Souvatzoglou M, Eiber M, et al. First clinical experience with integrated whole-body PET/MR: comparison to PET/CT in patients with oncologic diagnoses. J Nucl Med. 2012;53:845-855.

23. Wang Z, Ma Q, Li P, et al. Abberant expression of CXCR4 and beta-catenin in pancreatic cancer. Anticancer Res. 2013;33:4103-4110.

24. Bai S, Wang D, Klein MJ, Siegal GP. Characterization of CXCR4 expression in chondrosarcoma of bone. Arch Pathol Lab Med. 2011;135:753-758.

25. Gagliardi F, Narayanan A, Reni M, et al. The role of CXCR4 in highly malignant human gliomas biology: current knowledge and future directions. Glia. 2014;62: 1015-1023.

26. Portella L, Vitale R, De Luca S, et al. Preclinical development of a novel class of CXCR4 antagonist impairing solid tumors growth and metastases. PLoS One. 2013;8:e74548.

27. Fahham D, Weiss ID, Abraham M, et al. In vitro and in vivo therapeutic efficacy of CXCR4 antagonist BKT140 against human non-small cell lung cancer. $J$ Thorac Cardiovasc Surg. 2012;144:1167-1175.

28. Shim H, Lau SK, Devi S, Yoon Y, Cho HT, Liang Z. Lower expression of CXCR4 in lymph node metastases than in primary breast cancers: potential regulation by ligand-dependent degradation and HIF-1alpha. Biochem Biophys Res Commun. 2006;346:252-258.

29. Rosen JM, Jordan CT. The increasing complexity of the cancer stem cell paradigm. Science. 2009;324:1670-1673.

30. O'Brien CS, Howell SJ, Farnie G, Clarke RB. Resistance to endocrine therapy: are breast cancer stem cells the culprits? J Mammary Gland Biol Neoplasia. 2009;14: 45-54.

31. Dubrovska A, Hartung A, Bouchez LC, et al. CXCR4 activation maintains a stem cell population in tamoxifen-resistant breast cancer cells through AhR signalling. Br J Cancer. 2012;107:43-52.

32. Kucia M, Reca R, Miekus K, et al. Trafficking of normal stem cells and metastasis of cancer stem cells involve similar mechanisms: pivotal role of the SDF-1CXCR4 axis. Stem Cells. 2005;23:879-894.

33. Yang G, Xue F, Chen X. Prognostic value of different amounts of cancer stem cells in different molecular subtypes of breast cancer. Gland Surg. 2012;1:20-24.

34. Maurer T, Beer AJ, Wester H-J, Kübler H, Schwaiger M, Eiber M. Positron emission tomography/magnetic resonance imaging with 68-gallium-labeled ligand of prostate-specific membrane antigen: promising novel option in prostate cancer imaging? Int J Urol. 2014;21:1286-1288. 